\title{
Perception, Attitude et Attentes des Résidents à l'égard des Espaces Verts Urbains de Yamoussoukro (Côte d'Ivoire)
}

\author{
Kouassi Roland Hervé, \\ Laboratoire de Biologie Végétale, Département des Sciences et \\ Technologies, École Normale Supérieure d'Abidjan, Côte d'Ivoire \\ Nomel Gnagne Jules Richard, \\ Kouadio Yao Jean-Clovis, \\ Laboratoire de Botanique, UFR Biosciences, \\ Université Félix Houphouët-Boigny, Côte d'Ivoire, Abidjan, Côte d'Ivoire
}

Ambé Alain Serge Augustin,

Laboratoire de Biologie Végétale, Département des Sciences et

Technologies, École Normale Supérieure d'Abidjan, Côte d'Ivoire

N'guessan Kouakou Edouard,

Laboratoire de Botanique, UFR Biosciences,

Université Félix Houphouët-Boigny, Côte d'Ivoire, Abidjan, Côte d'Ivoire

Doi: 10.19044/esj.2019.v15n3p389 URL:http://dx.doi.org/10.19044/esj.2019.v15n3p389

\section{Résumé}

Les forêts urbaines considérées comme gênant par le passé font aujourd'hui l'objet d'une attention particulière, pour le bien-être de la population urbaine. Cette étude a pour objectif de recenser les avis des usagers des forêts urbaines de Yamoussoukro. Ainsi pour mener à bien cette étude, un questionnaire a été adressé aux usagers en vue de recueillir leurs avis sur les forêts urbaines de Yamoussoukro. Ce questionnaire a été complété avec des observations directes sur le terrain pour apprécier le comportement de la population à l'égard de ces espaces verts. Cette enquête a porté essentiellement sur la fréquentation de la population au niveau des arbres d'alignement. Au total, 284 usagers des espaces verts ont été interrogés de manière aléatoire. Ces usagers sont en majorité des hommes $(63,7 \%)$. Ils fréquentent ces espaces verts pour l'ombrage, le repos et pour y mener des activités. Selon ces usagers, les plantes embellissent les villes (14,6\%). Ensuite elles interviennent dans la régulation du climat (14\%), puis servent à se soigner $(10,3 \%)$, à se nourrir $(10,2 \%)$, etc. Le manque d'arbres sur certaines voies pour profiter de l'ombrage et l'absence de jardin public pour profiter de leurs aménités a amené $91,5 \%$ des enquêtés à souhaiter 
l'augmentation des arbres d'alignement et la création de jardin public. Ces résultats révèlent que les espaces verts jouent un rôle très important en milieu urbain et méritent donc une attention particulière des autorités administratives et politiques. Cependant les usagers affirment que la population elle-même n'entretient pas les espaces verts $(71,6 \%)$ au vue des actes qu'elle pose. Cela se manifeste par les coupes abusives des arbres, le prélèvement de leurs écorces ou le dépôt de déchets à leurs pieds. Ces résultats permettront aux autorités locales et administratives de prendre en compte ces espaces dans la planification et le développement de la ville pour le bien-être de la population.

Mots clés: Espaces verts urbains, attitude, perception, attente, Yamoussoukro, Côte d'Ivoire

\title{
Perception, Attitude and Expectations of Residents Towards Urban Green Spaces in Yamoussoukro (Côte d'Ivoire)
}

\author{
Kouassi Roland Hervé, \\ Laboratoire de Biologie Végétale, Département des Sciences et \\ Technologies, École Normale Supérieure d'Abidjan, Côte d'Ivoire \\ Nomel Gnagne Jules Richard, \\ Kouadio Yao Jean-Clovis, \\ Laboratoire de Botanique, UFR Biosciences, \\ Université Félix Houphouët-Boigny, Côte d'Ivoire, Abidjan, Côte d'Ivoire
}

Ambé Alain Serge Augustin,

Laboratoire de Biologie Végétale, Département des Sciences et

Technologies, École Normale Supérieure d'Abidjan, Côte d'Ivoire

N'guessan Kouakou Edouard,

Laboratoire de Botanique, UFR Biosciences,

Université Félix Houphouët-Boigny, Côte d'Ivoire, Abidjan, Côte d'Ivoire

\begin{abstract}
Urban forests that were once considered a nuisance are now being paid particular attention for the well-being of the urban population in contact with them. The objective of this study is to survey the opinions of the users of Yamoussoukro's urban forests. This questionnaire was completed with direct observations on the ground to assess the behavior of the population with regard
\end{abstract}


to these green spaces. To carry out this study, a questionnaire was sent to users to collect their opinions on the urban forests of Yamoussoukro. This survey focused on population attendance at the level of alignment trees. A total of 284 users of green spaces were randomly questioned. The majority of these users are male $(63.7 \%)$. They use these green spaces for shade, rest and the activities they offer. According to these users, first plants beautify cities (14.6\%). Next, they contribute to climate regulation (14\%), and are then used for health care $(10.3 \%)$ and food (10.2\%). The lack of trees on some roads to take advantage of the shade and the absence of a public garden to take advantage of their amenities led $91.5 \%$ of respondents to wish the increase of the trees of alignment and the creation of public garden. These results reveal that green spaces play a very important role in urban areas and therefore deserve a particular attention on the part of administrative and political authorities. However, users say that the population itself does not maintain green spaces $(71.6 \%)$ in view of the actions it takes. This is manifested by abusive cutting, waste generation and tree injury. These results will allow local and administrative authorities to take these spaces into account in the planning and development of the city for the well-being of the population.

Keywords: Green spaces, urban, users, perception, Yamoussoukro, Côte d'Ivoire

\section{Introduction}

Très longtemps, les forêts urbaines ont été négligées car elles ont été regardées comme "dérangeant" par les écologistes (Muratet et al., 2010). Mais de multiples études ont montré qu'elles sont une composante de l'environnement particulièrement importante au vue des nombreux services qu'elles rendent aux citadins. Les services rendus par les forêts urbaines sont diverses. Ils y a entre autre, l'augmentation de la valeur des propriétés (Sather et al., 2004), la réduction des surfaces d'érosion, de la consommation d'énergie (McPherson et al., 2007) et la réduction des frais de soin de santé, en raison de l'amélioration de la qualité de l'air (Nowak et al., 2006). Le lien entre les espaces verts et les citadins a contribué à leur développement dans la plupart des villes européennes, notamment Oslo, Bruxelles, Copenhague (Nilsson et Randrup, 2003). Dans les pays développés, la foresterie urbaine est très accentuée, principalement pour des raisons esthétiques et écologiques (Miller, 1997). Par contre dans les pays en développement et notamment en Afrique, la foresterie urbaine est à ses débuts (Simza, 2012). Cette discipline a vu le jour dans le souci de la préservation de ces espaces verts qui sont en net régression du fait de l'urbanisation grandissante. En effet selon les Nations Unies (2007), plus de la moitié de la population vit en ville depuis 2007, soit 3,3 milliards d'habitants. Cette situation, malheureusement rime avec la 
déforestation et la segmentation des habitats dans ce sens que le besoin d'espace et le développement économique induisent la surexploitation des ressources qui entrainent la régression et la disparition des espèces végétales et animales (Scheneiders et al., 2012).. Ainsi cette croissance exponentielle de l'urbanisation soulève des craintes relatives aux conséquences potentielles sur la conservation de la biodiversité et de la qualité du cadre de vie humaine (Dye, 2008). Les villes ivoiriennes et précisément Yamoussoukro, la capitale politique de la Côte d'Ivoire ne reste pas en marge de la dégradation des espaces verts urbains. Cette dégradation se manifeste par la régression et la disparition de ces espaces verts. Les processus d'urbanisation ont conduit à un accès inégal des espaces verts urbains et aux conséquences environnementales et sociales des avantages qui en découlent. Cette perte de la biodiversité urbaine est faite fait au détriment de l'urbanisation galopante. Ces espaces sont en danger car de manière générale, rares sont les projets qui en ont pris la mesure et qui ont engagé une politique de préservation de ces espaces. De plus, le comportement des populations vis à vis de ces espaces constitue un frein au bon fonctionnement de ceux-ci. Les questions sont de savoir comment communiquer sur cette biodiversité de sorte à assurer sa pérennité ? Quels sont les rôles que jouent les forêts urbaines de Yamoussoukro dans la vie de la population? Quels sont les attitudes de la population à l'égard de ces espaces verts ? C'est dans cette optique que cette étude a été menée pour une meilleure connaissance et une préservation de ces espaces. Les objectifs de ce travail sont d'étudier les perceptions, les attitudes et les attentes de la population sur les espaces verts, et de collecter les informations sur les rôles des espaces verts urbains de Yamoussoukro.

\section{Matériel et Méthodes \\ Zone d'étude}

Yamoussoukro, capitale politique et administrative de la Côte d'Ivoire est la zone d'étude. Elle est située au centre de la Côte d'Ivoire entre $6^{\circ} 40^{\prime}$ et $7^{\circ}$ de latitude Nord et entre $5^{\circ} 10^{\prime}$ et $5^{\circ} 20^{\prime}$ de longitude Ouest. L'étude a été réalisée dans 7 quartiers de la ville de Yamoussoukro (220 logements, Assabou, Dioulakro, Kokrenou, Millionnaire, Morofé, Sopim) (Figure 1). Le choix de ces quartiers est dû au fait que ces quartiers disposent d'espaces verts fréquentés par la population. 


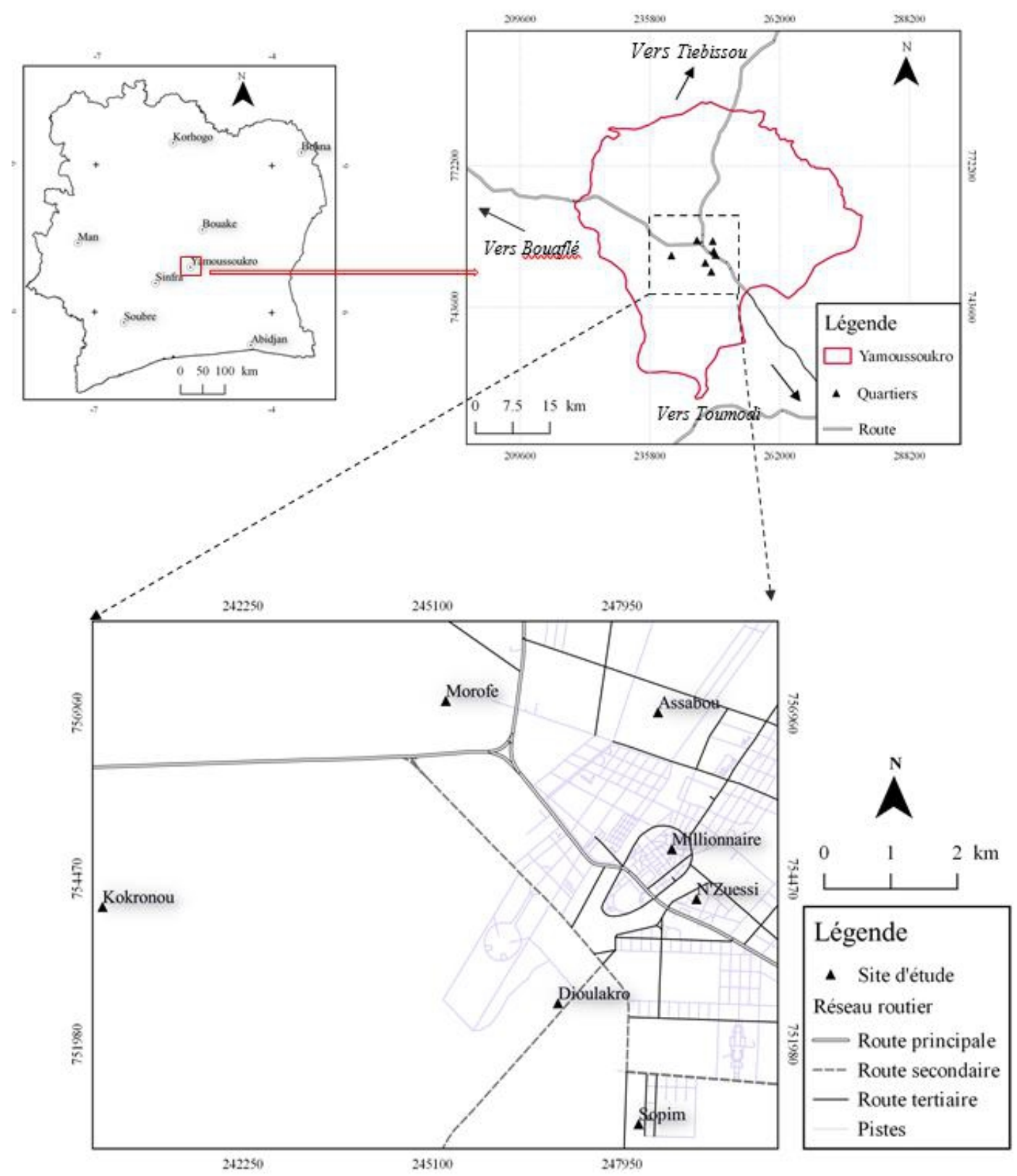

Figure 1: Cartes de localisation des sites d'étude dans le District autonome de Yamoussoukro

\section{Collecte des données}

Pour connaitre les perceptions, les attitudes et les attentes des populations sur les forêts urbaines de Yamoussoukro, une enquête sociologique a été menée. Un questionnaire a permis de renseigner 
premièrement les raisons de la fréquentation des usagers sur ces espaces verts. Deuxièmement, il a permis de connaître les perceptions qu'ont les enquêtés sur les espaces verts urbains à travers les différents rôles que jouent les plantes dans leur environnement et les problèmes qu'ils peuvent poser. Troisièmement, il a permis de connaître leur attitude vis-à-vis à de ces espaces verts à travers leur comportement et enfin leurs attentes en matière de foresterie urbaine. Un guide d'entretien a aussi été adressé aux autorités locales dont un agent de la mairie, un agent du district autonome et un agent de la pépinière d'Etat de Yamoussoukro. En plus des entretiens avec les usagers et les autorités, des observations directes ont été faites sur le terrain afin d'apprécier l'état des espaces verts et le comportement de la population à leur égard. La méthode d'échantillonnage stratifiée non probabiliste a été utilisée. Elle a consisté à diviser la zone d'étude en différentes strates et à y associer un nombre différents d'enquêtés. Dans ce travail, chaque quartier a constitué une strate. Dans chacune de ces strates, un espace vert a été visité et des utilisateurs de ces espaces verts ont été interviewés de manière aléatoire. L'enquête a porté essentiellement sur les arbres d'alignement, le seul type d'espace vert existant dans la ville de Yamoussoukro. Au total, 284 usagers des espaces verts (notamment les arbres d'alignement) ont été interrogés (Tableau 1).

Tableau 1:Répartition des enquêtés par strate

\begin{tabular}{ccc}
\hline Strates & Zones (Quartiers) & nombres cités \\
\hline 1 & N'Zuessi & 50 \\
2 & Assabou & 50 \\
\hline 3 & Dioulakro & 50 \\
\hline 4 & Kokrenou & 32 \\
\hline 5 & Millionnaire & 50 \\
\hline 6 & Morofé & 30 \\
\hline 7 & Sopim & 22 \\
\hline TOTAL & & $\mathbf{2 8 4}$
\end{tabular}

\section{Analyse de données}

A partir des variables Collectées, notamment le profil de chaque enquêté (sexe, âge, niveau d'étude, profession), nous avons pu caractériser les usagers des espaces verts de notre zone d'étude.

Le test de $\mathrm{Khi}^{2}$ a été utilisé dans cette étude pour comparer les pourcentages des différentes variables mises en relation à l'aide du logiciel Sphinx Plus ${ }^{2}$ version 4.5. Lorsque le lien existe entre les variables étudiées, 
nous avons utilisé l'AFC pour les discriminer. La plus petite différence significative a été fixée pour $\mathrm{p}<0,0001$.

\section{Résultats}

\section{Profil des enquêtés}

103 femmes et 181 hommes soit respectivement 36,3\% et 63,7\%. La plupart de la population enquêtée a un âge compris entre 21 et 30 ans avec une proportion de 24,6\% suivi de ceux ayant un âge compris entre 31 et 40 ans avec une proportion de $24,4 \%$. Les moins nombreux sont ceux ayant moins de 10 ans. La majorité des personnes interrogées ont le niveau secondaire soit $53,9 \%$. Les non scolarisés arrivent en dernière position avec une proportion de 10,2\%. Au niveau de la profession des enquêtés, 30,6\% des usagers des espaces verts sont des commerçants. Les retraités et la catégorie "'autres'" constituée des sans-emplois et des ménagères ont les plus faibles pourcentages avec respectivement 2,5 et $1,8 \%$ (Tableau 2). Les différences entre les différentes proportions sont significatives.

Tableau 2: Récapitulatif du profil des enquêtés ( $\mathrm{N}=284)$

\begin{tabular}{|c|c|c|c|}
\hline $\begin{array}{l}\text { Variables du } \\
\text { profil }\end{array}$ & $\begin{array}{l}\text { Nombre de } \\
\text { citation }\end{array}$ & Pourcentages (\%) & Tests statistiques \\
\hline Genre & & & \multirow{3}{*}{$\begin{array}{c}\text { Chi2 }=21,45 \\
\text { p }<0,0001\end{array}$} \\
\hline Féminin & 103 & $36,3^{\mathrm{a}}$ & \\
\hline Masculin & 181 & $63,7^{\mathrm{b}}$ & \\
\hline Age & & & \multirow{8}{*}{$\begin{array}{c}\text { Chi2 }=110,37 \\
\text { p }<0,0001\end{array}$} \\
\hline $0-10$ & 01 & $0,4^{\mathrm{a}}$ & \\
\hline $11-20$ & 48 & $16,9^{\mathrm{b}}$ & \\
\hline $21-30$ & 70 & $24,6^{\mathrm{c}}$ & \\
\hline $31-40$ & 69 & $24,3^{\mathrm{c}}$ & \\
\hline $41-50$ & 56 & $19,7^{b}$ & \\
\hline $51-60$ & 27 & $9,5^{\mathrm{d}}$ & \\
\hline 61 et plus & 13 & $4,6^{\mathrm{e}}$ & \\
\hline \multirow{2}{*}{\multicolumn{3}{|c|}{$\begin{array}{c}\text { Niveau } \\
\text { d'instruction }\end{array}$}} & \multirow{6}{*}{$\begin{array}{c}\mathrm{Chi}^{2}=138,64 \\
\mathrm{P}<0,0001\end{array}$} \\
\hline & & & \\
\hline Non scolarisé & 29 & $10,2^{\mathrm{a}}$ & \\
\hline Primaire & 67 & $23,6^{b}$ & \\
\hline Secondaire & 153 & $53,9^{c}$ & \\
\hline Supérieur & 35 & $12,3^{\mathrm{a}}$ & \\
\hline \multicolumn{3}{|l|}{ Profession } & \multirow{7}{*}{$\begin{array}{c}\mathrm{Chi}^{2}=120,92 \\
\mathrm{P}<0,0001\end{array}$} \\
\hline Commerçants & 87 & $30,6^{\mathrm{a}}$ & \\
\hline Artisans & 53 & $18,7^{\mathrm{b}}$ & \\
\hline Salariés & 67 & $23,6^{\mathrm{c}}$ & \\
\hline élèves et étudiants & 65 & $22,9 c$ & \\
\hline Retraités & 7 & $2,5^{\mathrm{d}}$ & \\
\hline Autres & 5 & $1,8^{\mathrm{d}}$ & \\
\hline
\end{tabular}

Légende : Sur une même ligne les moyennes portant les mêmes lettres sont statistiquement égales. 


\section{Usages des espaces verts de Yamoussoukro}

Les raisons de fréquentation des espaces verts sont multiples. La raison principale de la présence des usagers à ces endroits est l'ombrage $(22,7 \%)$. Les raisons secondaires citées sont diverses. Les enquêtés utilisent ces espaces pour se reposer $(20 \%)$, se restaurer $(13,8 \%)$, exercer leur métier $(12,2 \%)$ (le commerce, la mécanique, l'artisanat), pour réfléchir $(10,6 \%)$ et pour se distraire (10,3\%). Moins de 6\% des usagers utilisent les espaces verts respectivement pour des rencontres $(4,4 \%)$, les études $(3,5 \%)$ et les prières $(2,5 \%)$ (Figure 2).

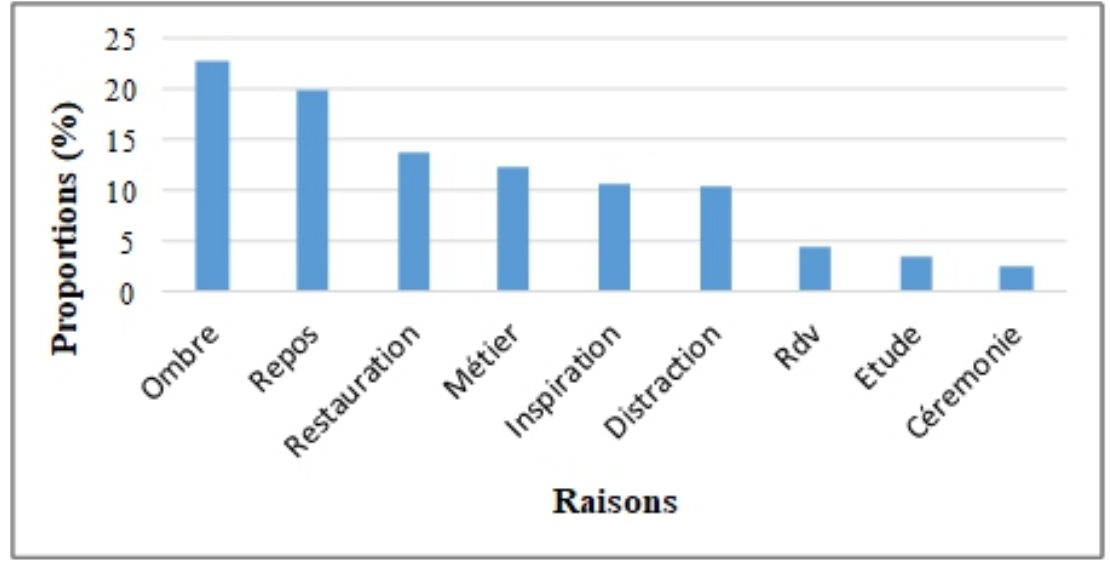

Figure 2: Répartition des raisons de présence des enquêtés sur ces espaces verts urbains

Lorsqu'on considère le niveau d'étude et les usages des espaces verts, le test de $\mathrm{Chi}^{2}$ montre qu'il existe un lien statistique entre ces deux variables. En effet, les usagers ayant le niveau primaire et les non scolarisés utilisent les espaces verts dans le cadre de leur métier et cérémonie. Ceux ayant le niveau secondaire fréquentent ces espaces pour le repos, la distraction, les études et la restauration. Les usagers ayant le niveau supérieur les fréquentent pour le repos, la distraction, les rencontres et les études (Figure 3).

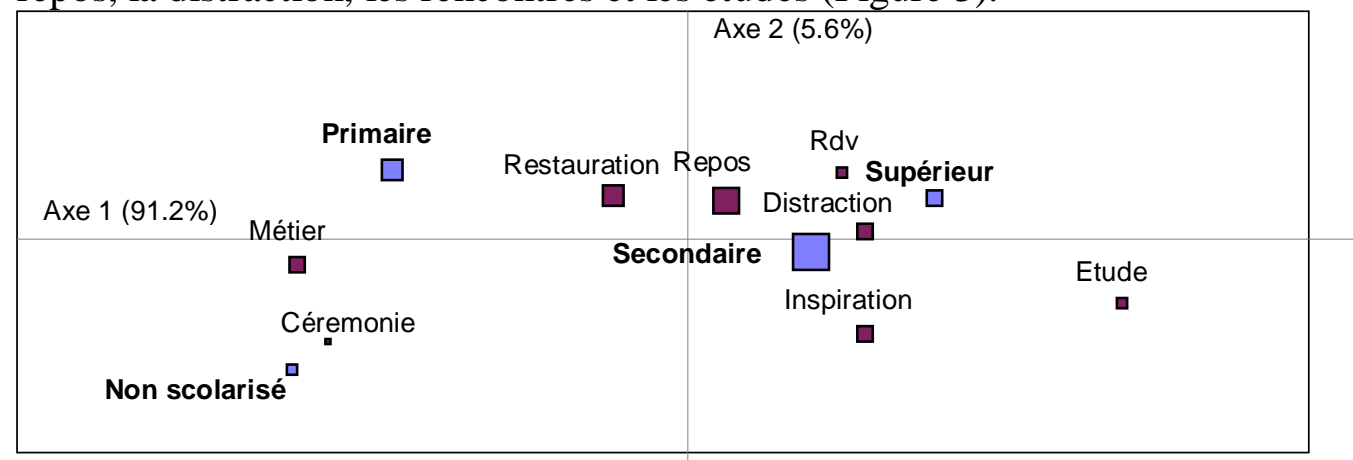

Chi2 $=96,03 ; \mathrm{ddl}=21 ; \mathrm{p}=0,0001$

Figure 3: Relation entre le niveau d'étude et les raisons de la présence des usagers sur les espaces verts urbains 


\section{Perception des enquêtés sur les biens et services rendus par les espaces verts urbains}

La majorité des enquêtés estiment que les espaces verts sont importants dans la vie quotidienne au vu des nombreux services qu'ils rendent. Selon les enquêtés, les espèces végétales servent à l'embellissement $(14,6 \%)$ des rues et des propriétés, permettent de réguler le climat (14\%), de se soigner $(10,2 \%)$, de se nourrir $(10,2 \%)$ et servent de combustible (10,2\%). De même, les arbres sont le lieu de petits métiers $(9,4 \%)$. La catégorie autre $(0,3 \%)$ renferme les rôles cités rarement tels que les plantes constituent des barrières, chassent les mauvais esprits (Figure 4).

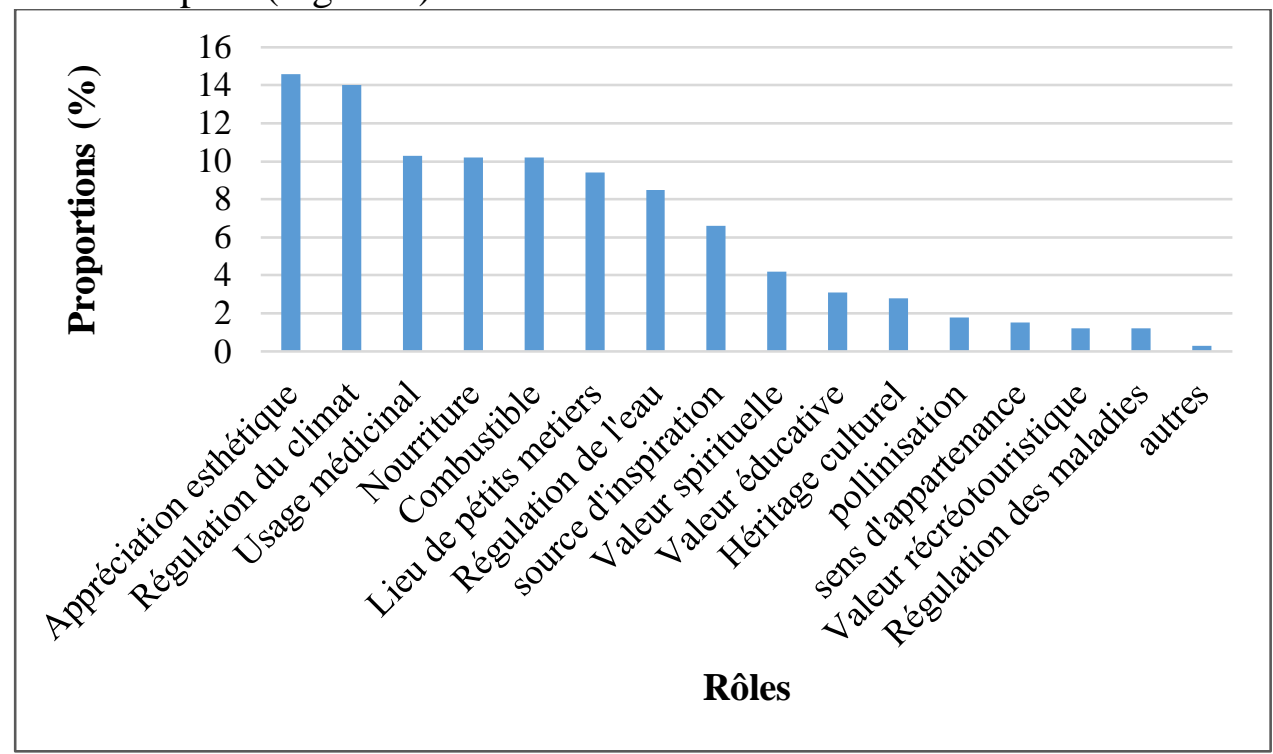

Figure 4: Connaissances des rôles joués par les espèces végétales en ville

Le test de $\mathrm{Chi}^{2}$ a montré le lien existant entre le niveau d'étude et les perceptions des usagers des espaces verts. Les enquêtés ayant le niveau primaire et les non scolarisés apprécient les espaces parce qu'ils fournissent de la nourriture, des plantes médicinales, et des fibres ligneuses combustibles. La présence des arbres permettent selon les usagers ayant le niveau supérieur, de purifier l'air, de réguler le climat, l'eau, l'érosion et les maladies. Ceux ayant le niveau secondaire les apprécient parce qu'en plus des fonctions citées, les arbres permettent de trouver une source d'inspiration et sont le lieu de petits métier (Figure 5). 


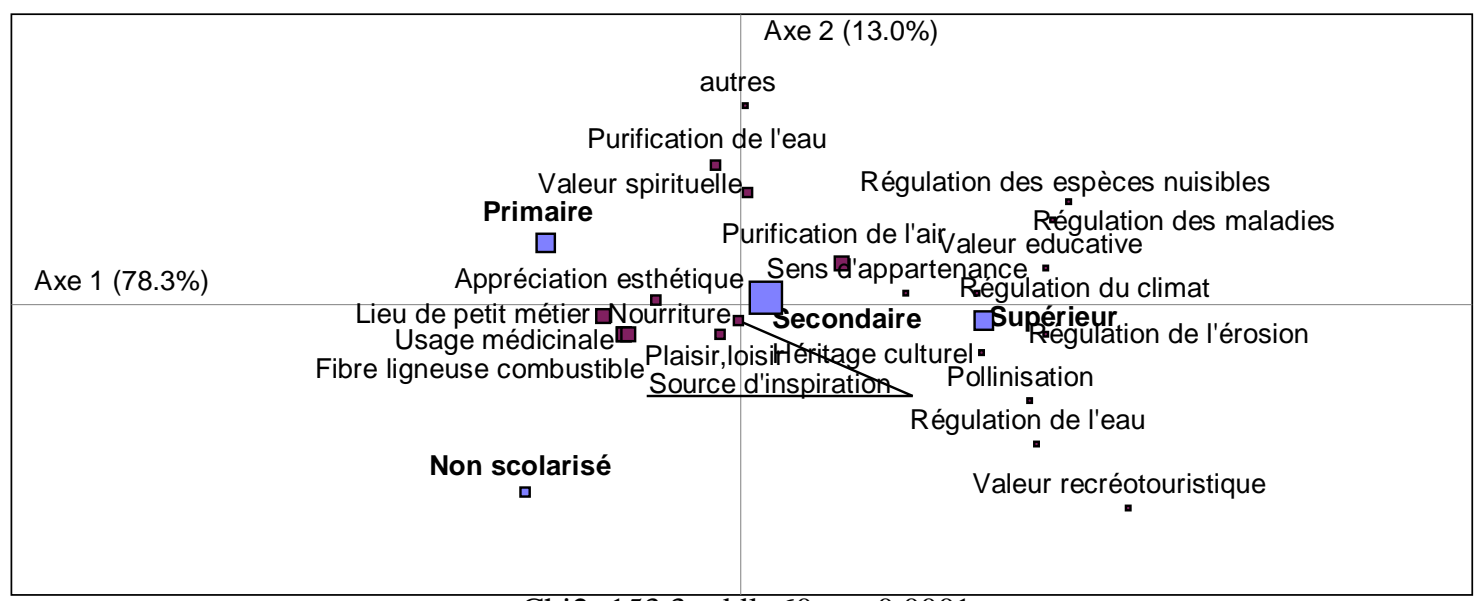

Chi2 $=153,3 ; \mathrm{ddl}=60 ; \mathrm{p}=0,0001$

Figure 5: Relation entre le niveau d'étude et les perceptions des enquêtés des espaces verts urbains

Concernant les usages potentiels faits des arbres, les usages alimentaires $(32,6 \%)$ et médicinaux $(31,5 \%)$ sont les plus cités. La Catégorie 'autre' (2,6\%) renferme la confection de nattes, de balai, la chasse des mauvais esprits sont citées rarement (Figure 6).

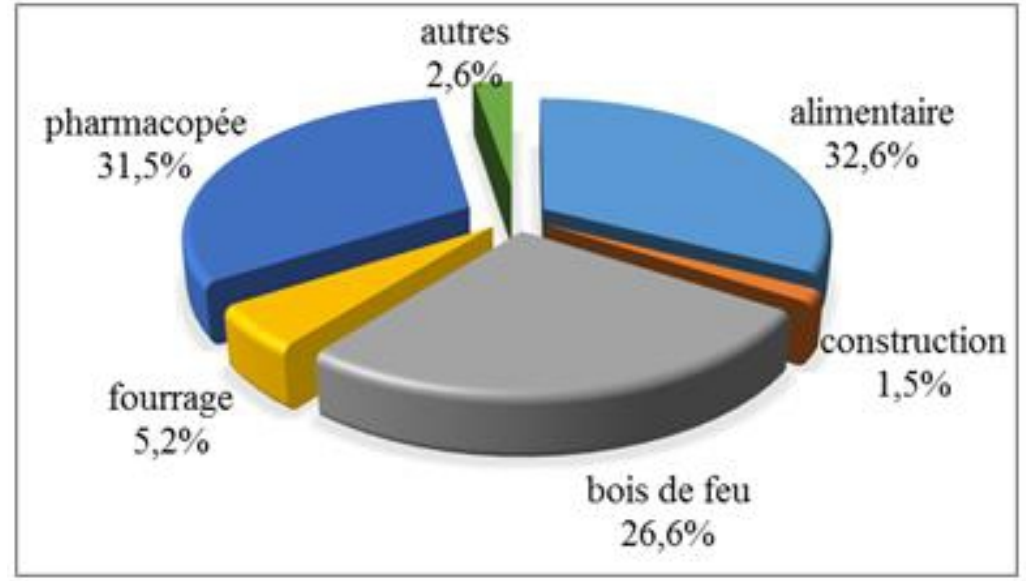

Figure 6 : Usages potentiels faits des arbres en ville

Selon les usagers, les espaces verts ont aussi des inconvenants. Les arbres qui y sont plantés sont par les feuilles, les branches et les graines qui tombent, salissants $(29,7 \%)$. Ensuite ils attirent les moustiques, les insectes et les reptiles $(26,8 \%)$. Ils détruisent les voies $(21,3 \%)$ par leur proximité avec le bitume et menacent les bâtiments $(8,1 \%)$.Ils constituent aussi une cachette pour les malfaiteurs $(3,6 \%)$ (Figure 7 ). 


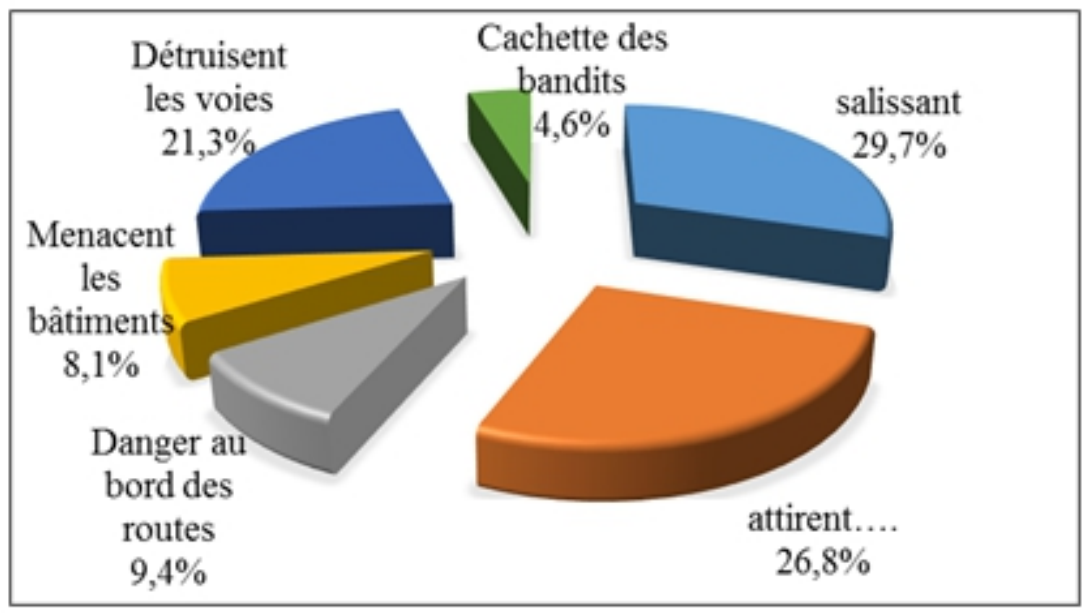

Figure 7: Les inconvénients liés à la présence espaces verts en ville

\section{Attitude de la population vis-à-vis des espaces verts urbains}

Les observations directes sur le terrain ont montré que les espaces verts urbains ne sont pas entretenus. Cela se traduit par les feuilles, les branches et les graines mortes qui jonchent les trottoirs. Aussi les espaces verts notamment «les bois » sont des toilettes à ciel ouvert pour la population qui y viennent pour leurs besoins. Les coupes abusives d'arbres pour diverses utilisations sont des signes qui montrent l'incivisme de la population à l'égard des espaces verts. Cette dans cette même optique que $71,6 \%$ des enquêtés ont affirmé que la population ne participe pas à l'entretien des espaces verts urbains contre $28,4 \%$ qui affirment au contraire que la population participe à l'entretien des espaces verts y compris eux-mêmes. Les $71,6 \%$ ayant affirmé que la population ne participe pas à l'entretien de ces espaces verts et cela se traduit de la façon suivante: $34,9 \%$ ont affirmé que la population produit des déchets et $36,7 \%$ prélèvent ou coupent abusivement les arbres (Figure 8 ).

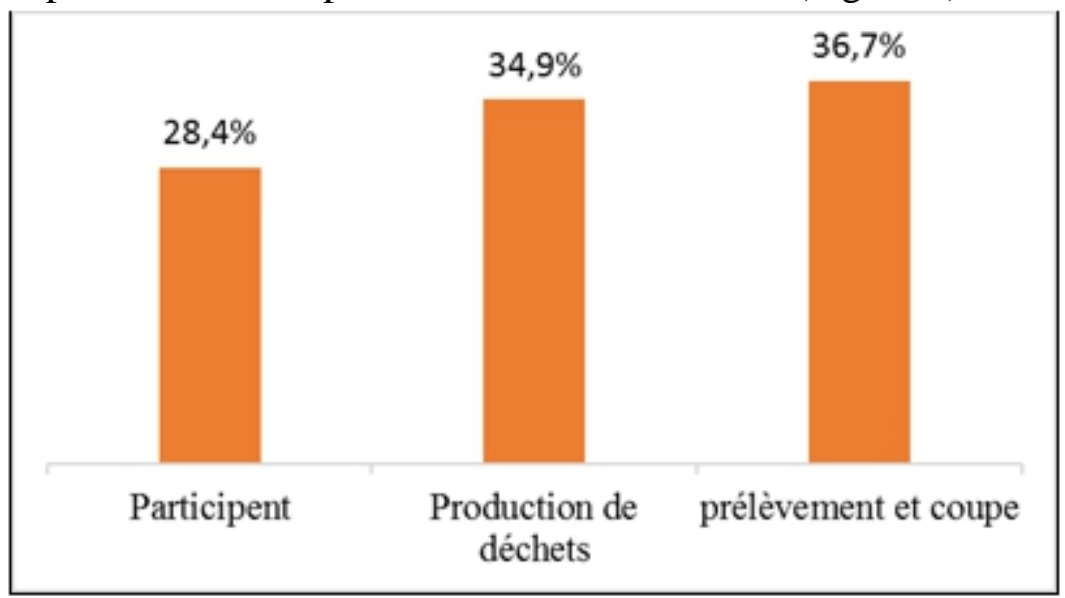

Figure 8: Répartition de la participation des enquêtés à l'égard des espaces verts 


\section{Attente des populations à l'égard des espaces verts}

Les usagers trouvent insuffisant les arbres d'alignement présents dans la ville de Yamoussoukro et a souhaité à 91,5\%, l'augmentation de ceux-ci. Les espèces souhaitées pour le reboisement des voies sont pour la plupart des espèces d'ombrage $(66,8 \%)$; elles sont suivies des espèces ornementales $(18,6 \%)$ et des espèces alimentaires $(13,4 \%)$. Les espèces médicinales $(1,23 \%)$ arrivent en dernière position (Figure 9). Sur les 284 personnes interrogées, 280 ont souhaité la création de jardins publics soit $98,6 \%$ contre 4 personnes qui n'ont pas voulu de jardins publics soit 1,4\% (Figure 10).

Les $98,6 \%$ des usagers souhaitant la création de jardin public ont donné les raisons suivantes: La première raison évoquée est que ces espaces offrent du repos (26,5\%). Ensuite les jardins permettent de se distraire (24,2\%). Les raisons les moins citées sont les usages cérémonies $(1,5 \%)$ et air frais $(1,9 \%)$ (Figure 11).

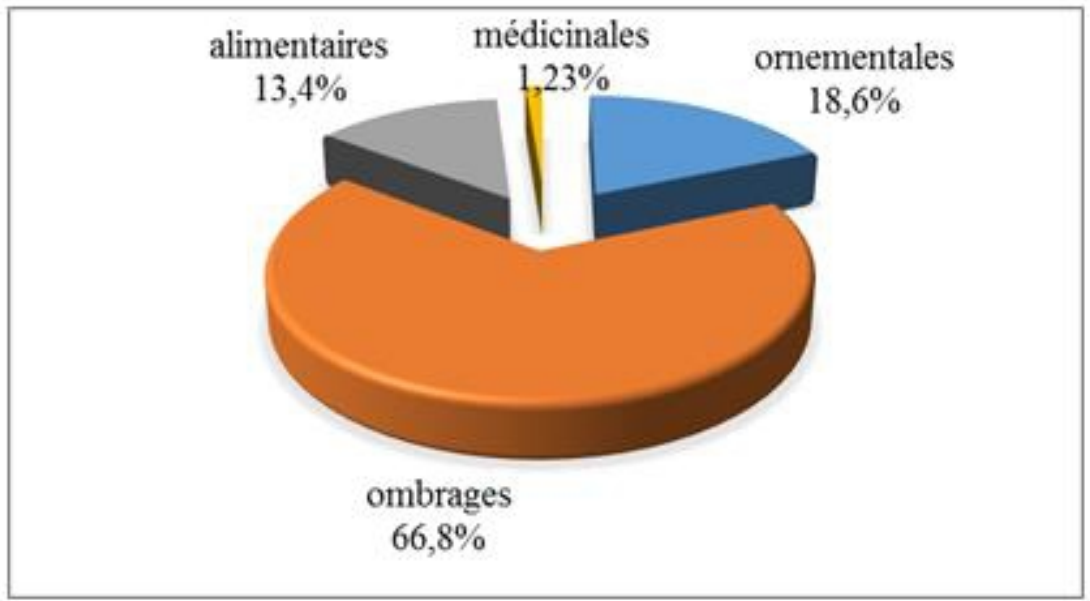

Figure 9: Répartition des espèces souhaitées pour l'enrichissement des voies

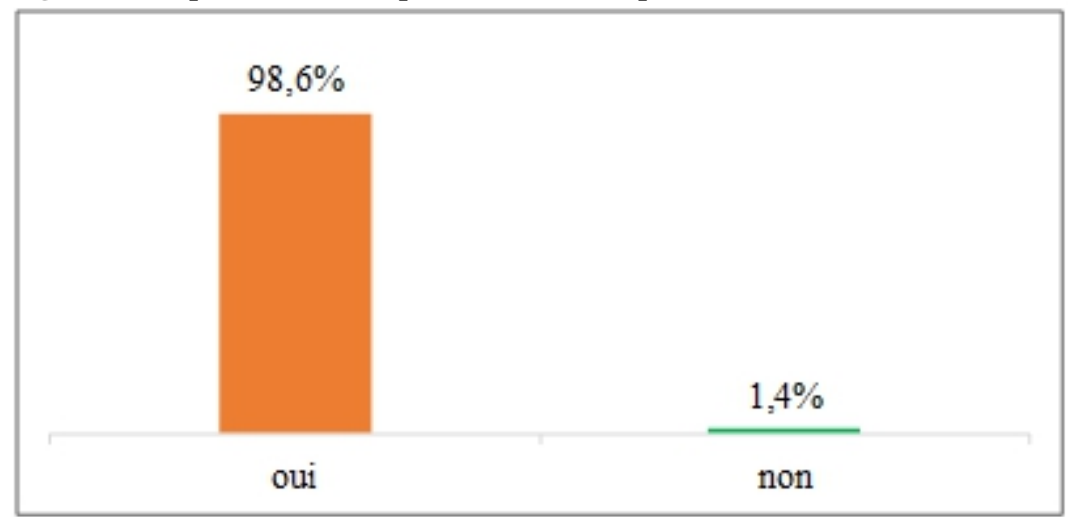

Figure 10: Répartition de l'acceptation de la création de jardin public 


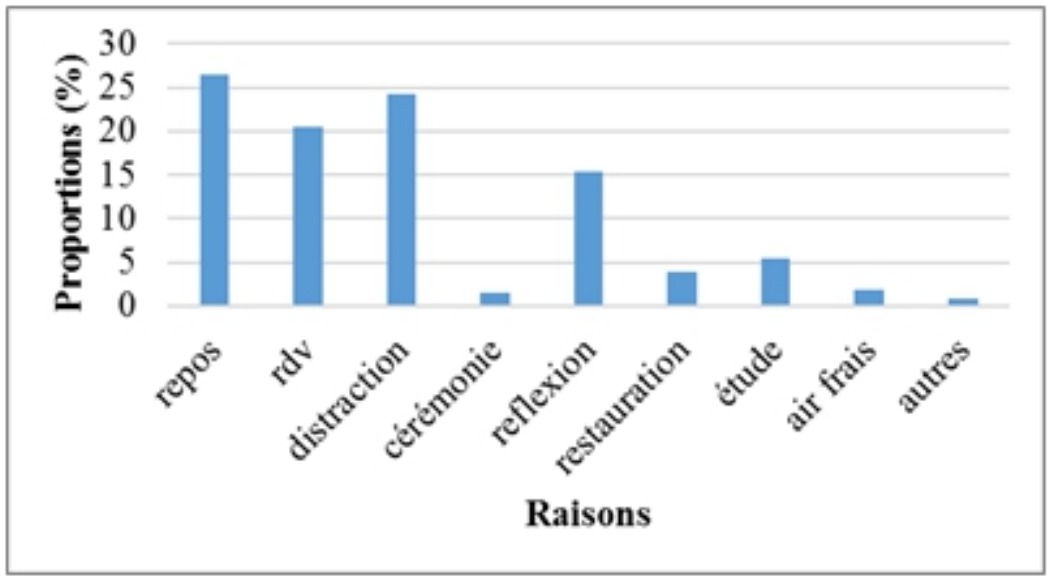

Figure 11: Raisons pour la création de jardins publics

\section{Discussion}

Les espaces verts de la ville de Yamoussoukro à l'unanimité ont été reconnus importants par les personnes enquêtées en raison de leurs nombreuses utilités. La raison principale de la présence des enquêtés sur ces espaces est l'ombrage que procure les arbres urbains. Cela s'explique par le fait qu'à Yamoussoukro, les températures sont élevées (températures diurnes toujours supérieures à $30^{\circ} \mathrm{C}$ ), et les populations trouvent la fraicheur à l'ombre des arbres. Ces résultats sont similaires à ceux obtenus par Kouadio (2016) dans les jardins du plateau et de Cocody et Monssou et al. (2016) dans le jardin botanique et de Dalhia fleur de Bingerville. La présence accrue d'arbres dans les rues permettra aux populations de profiter d'un maximum d'ombrage lors des marches, des attentes et des rencontres. Selon Heisler (1986), l'arbre crée un microclimat qui est très prisé par la population; donc très important (AEE, 2012). Les enquêtés utilisent les espaces verts pour exercer leur métier. Compte tenu de la proximité des arbres au bord des voies, ceux-ci offrent un endroit très fréquenté par les piétons et les automobilistes et un endroit propice aux différentes activités commerciales. Selon Nagendra et Gopal (2009), les arbres fournissent des abris aux vendeurs de rue. Les élèves et étudiants vont sur les espaces verts pour faire leurs travaux scolaires car ces lieux sont calmes et apaisants. Taylor et al. (2001) ont démontré que le contact régulier avec un espace vert entraine une hausse de l'attention et la discipline des enfants à l'école. Les espaces verts sont appréciés parce qu'ils embellissent les rues et font plaisir à contempler. Ils brisent la monotonie des surfaces minéralisées. Ainsi les espaces verts et les arbres sont attachés à la qualité de vie (Wolf, 2003). Concernant les perceptions, elles sont de deux ordres. Les perceptions positives qui concernent les différents rôles que jouent les espaces verts et les perceptions négatives qui sont les problèmes que cause la présence de ces espaces en milieu urbain. Dans cette étude, les perceptions positives (16) sont 
plus nombreuses que les perceptions négatives (6). La perception négative la plus citée est que les espaces verts salissent. Cela est dû au fait que les feuilles et les branches qui tombent sur les trottoirs ou dans les rues rendent ces endroits non fréquentables. Cela se fait ressentir lorsqu'il y a absence d'entretien et la fréquence de chute des feuilles et des branches quotidiennes rendent l'entretien assez pénible. Ce problème a été évoqué par certains auteurs tels que Kouadio (2016) et Nomel (2016) à Abidjan et Hunter (2001) en Finlande dans leur étude en milieu urbain. Par contre l'étude menée à Guangzhou en Chine a montré que la population ne considère pas la saleté engendrée par la litière organique comme un problème dans le sens où les espaces verts restent éloignés des habitations et que les arbres ont des feuilles persistantes (Jim et Chen, 2006). Les espaces verts en milieu urbain ont été reconnus comme des lieux d'agression et de refuge des malfaiteurs. En effet, ces endroits non surveillés et mal éclairés sont des zones à risque pour les automobilistes et les passants qui s'aventurent sur les voies qui traversent les espaces verts ou à proximité de ceux-ci. Des études similaires à Guangzhou ont montré que les espaces verts par leur obscurité constituent un problème pour la sécurité des résidents notamment des menaces de cambriolage (Jim et Chen, 2006). En majorité, la population n'entretient pas les espaces verts. Cela se traduit par les coupes abusives de plantes pour diverses utilisations, en grande partie comme bois de chauffe. Elle se traduit également par le fait que ces espaces verts non surveillés sont transformés en de véritables toilettes à ciel ouvert par la population. L'élagage des arbres d'alignement au profit des magasins a aussi été révélé par les autorités locales. La faible proportion de personnes participant à l'entretien des espaces verts s'explique par le fait que les personnes possédant une maison proche des espaces verts effectue un balayage régulier et un élagage de ces arbres pour ne pas que ceux-ci aient un inconvénient sur leur quotidien. Aussi les personnes utilisant les arbres d'alignement pour leurs activités les entretiennent. Au vu des nombreux rôles que jouent les arbres d'alignement, notamment la purification de l'air, l'ombrage, l'esthétique et au vu de l'insuffisance de la couverture en arbres d'alignement de la ville, les enquêtés et souhaitent leur augmentation. Ces résultats sont similaires à ceux de Polorigni et al. (2014) à Lomé. Les espèces d'ombrage sont les plus sollicitées pour l'enrichissement des voies. L'absence de jardin public dans la ville de Yamoussoukro a orienté sa population vers les arbres d'alignement afin de profiter des aménités liées à leur présence. Cette absence explique aussi le fait que les populations souhaitent vivement que des jardins publics soient aménagés dans la ville.

\section{Conclusion}

La présente étude a permis de recenser les perceptions, les attitudes et les attentes des usagers à l'égard des espaces verts urbains. Elle a permis de 
montrer que les espaces verts sont importants pour la population qui les fréquente. Les espaces verts en général et notamment les arbres d'alignement produisent de l'ombrage qui est très prisé par les usagers qui les fréquentent pour diverses raisons qui sont entre autres le repos, la distraction, les études, l'exercice de leur métier. Compte tenu des avantages liés à la présence des arbres notamment la régulation du climat, l'embellissement, le lieu de petits métier, etc., les usagers souhaitent l'augmentation des arbres de rue et la création de jardin public pour profiter des aménités liés à leur présence. Cependant Ces usagers ont affirmé que la population coupent les arbres et produisent des déchets sur les espaces verts ce qui favorise leur dégradation. Aussi les usagers soulignent le manque d'implication des autorités dans la gestion de ces espaces. Cela se traduit par les inconvénients tels que les agressions aux abords de ces espaces et la saleté causée par la chute des branches et feuilles. La création de jardins et la gestion des espaces verts peuvent être un moyen dans l'amélioration de la qualité de vie des populations en contact avec les espaces verts. Pour cela, des séances de sensibilisation auprès de la population et la formation à la gestion des espaces verts devraient être entreprises par les autorités locales pour le bien être de la population.

\section{References:}

1. Agence Européenne de l'Environnement. (2012). Etude sur l'impact du changement climatique en milieu urbain (vagues de chaleur, sécheresse, inondations), How vulnérable is your city ? (http://www.eea.europa.eu/hightlights/how-vulnerable-is-your-city).

$21 \mathrm{p} \quad$ Dye, C. (2008). Santé et vie urbaine. Science. 319, 766-769.

2. Heisler, G. M. (1986). Energy savings with trees. Journal of Arboriculture, 12(5): 113-125.

3. Hunter, I. R. (2001). What do people want from urban forestry? The Europe experience. Urban Ecosystems 5:277-284. Jim, C. Y., \& Chen, W. Y. (2006). Perception and Attitude of Residents Toward Urban Green Spaces in Guangzhou (China). Article in Environmental Management October 2006 Impact Factor: 1.72 DOI: 10.1007/s00267-005-0166-6 Source: PubMed. Environmental Management Vol. 38, No. 3, pp. 338-349.

4. Kouadio, Y. J. C. (2016). Diversité végétale, services écosystémiques et valeur économique de la végétation urbaine de la ville d'Abidjan (Côte d'Ivoire). Thèse de Doctorat UFHB. Laboratoire Botanique, Foresterie urbaine, 200 p.

5. McPherson, E. G., Simpson, J. R., Peper, P.J., Gardner, S. L., Vargas K. E., \& Xiao, Q. (2007). Northeast Community Tree Guide Benefits, Costs, and Strategic Planting, General Technical Report, 
PSW-GTR-202 [en ligne]. United States Department of Agriculture, Forest Service, Pacific Southwest Research Station, Center for Urban Forest Research, Davis, Californie, ÉtatsUnis, 106 p. www.fs.fed.us/psw/ (consultee le 23 Juillet 2018).

6. Miller, J. R. (1997). Urban forestry: Planning and management of green space. Prentice Hall, UpperSaddle River, New Jersey, pp. 22-67.

7. Monssou, E. O., Vroh, B. T. A., Goné, B. Z. B., Adou Yao, C. Y., \& N'Guessan, K. E. (2016). Evaluation de la diversité et estimation de la biomasse aérienne des arbres du jardin botanique de Bingerville (district d'Abidjan, Côte d'Ivoire), European Scientific Journal 12 (6) : $185-201$.

8. Muratet, Audrey., Porchet, E., Devictor, V., Arnal, G., Moret, J., Wright, S. , \& Machon, N. (2008). -Evaluation of floristic diversity in urban areas as a basis for habitatmanagement. Applied Vegetation Science 11: 451-460, 2008 doi: 10.3170/2008-7-18530, published online 4 June 2008. (O IAVS; Opulus Press Uppsala pp 451460.

9. Nations Unies. (2007). World urbanization prospects. New York, United organization.

10. Nagendra, H., \& Gopal, D. (2009). Street trees in Bangalore: Density, diversity,composition and distribution. Urban Forestry \& Urban Greening,journal homepage: .elsevier.de/ufug, $9 \mathrm{p}$.

11. Nilsson, K., \& Randrup T.B. (2009). Crise de la nature dans l'agglomération abidjanaise: L'exemple de la colonisation des espaces verts par l'habitat et les commerces dans la commune de Cocody, Abidjan, Côte d'Ivoire, $10 \mathrm{p}$.

12. Nomel, G. J. R. (2016). Diversité des espèces végétales et services écosystémiques des espaces aménagés des échangeurs de l'autoroute Cocody-Yopougon (Abidjan), 162, Mémoire de Master, UFR Biosciences, Université Félix HOUPHOUËET-BOIGNY, Côte d'Ivoire, $61 \mathrm{p}$.

13. Nowak, D. J., Crane, D.E., \& Stevens J.C. (2006). Air Pollution Removal by Urban Trees and Shrubs in the United States. Urban Forestry \& Urban Greening, 4: 115-123. Polorigni, B., Raoufou, R., \& Kouami, K. (2014). Perceptions, tendances et préférences en foresterie urbaine: cas de la ville de Lomé au Togo. European Scientific Journal 10 (5): 1857-7431.

14. Sather, I., Macie, E., \&. Hartel, D. R. (2004). Benefits and costs of the urban forest. In: Urban Forestry Manuel (c), USDA Forest Service, Athens, Georgia, États-Unis, 27p.

15. Schneiders, A., Van Daele, T., Van Landuyt, W., \& Van Reeth ,W. (2012). Biodiversity and ecosystem services: Complementary 
approaches for ecosystem management? Ecological Indicators 21 : 123-133.

16. Simza, D. (2012). La foresterie urbaine et sa contribution à la séquestration du carbone: Cas de la ville de Lomé (Togo). Mémoire de DEA de biologie de développement, Université de Lomé, Togo, 55

17. Taylor, A. F., Kuo, F. E. \& Sullivan, W. C. (2001). Coping with ADD: The

18. Surprising Connection to Green Play Settings. Environment Behavior, 33(1): 54-77.

19. Wolf, K . L. (2003). Social Aspects of Urban Forestry Public Response to the Urban Forest in Inner City Business Districts. Journal of Arboriculture 29 (3): 117-126. 